\title{
Prise en charge odontologique d'une patiente atteinte d'un angioedème héréditaire de type I
}

\section{Moizan H, Croze J, Marie I, Lévesque H. (Hôpital Saint Julien Service d'Odontologie, CHU de Rouen)}

L'œdème angio-neurotique héréditaire $(\mathrm{OAH})$ est une maladie orpheline génétique à transmission autosomique dominante, susceptible d'engager le pronostic vital par le biais d'un œdème laryngé fatal et de se manifester par des signes semblables à une occlusion digestive (Longhurst et al. 2012)

Les crises d'œdème récurrentes sont favorisées par des traumatismes tissulaires minimes, l'infection et autres facteurs déclenchants (prise d'un contraceptif oral, d'un inhibiteur de l'enzyme de conversion de l'angiotensine, période de stress, ....). L'étiologie de cette affection est un déficit qualitatif ou quantitatif en inhibiteur de la fraction C1 du complément (C1 inh). Le diagnostic biologique est confirmé par un taux sérique de $\mathrm{C} 4$ bas et de $\mathrm{C} 1$ inh très abaissé voire nul (Bouillet et al. 2011 ; Floccard et al. 2011 ; Bowen et al. 2010).

Les soins dentaires chirurgicaux et/ou conservateurs sont considérés comme de possibles facteurs initiateurs d'une crise d'angio-oedème caractérisée par un œdème blanc, mou, déformant, circonscrit à une région faciale, non prurigineux, ne répondant pas aux corticoïdes, ni aux antihistaminiques, et pouvant apparaitre d'emblée au niveau laryngé (Bork et al. 2011). Cette dernière localisation est la forme la plus grave et imprévisible avec une symptomatologie à type de dysphagie, de dysphonie puis de détresse respiratoire avec, en l'absence de traitement, un risque de décès en une vingtaine de minutes. La littérature relate des cas mortels à la suite d'avulsions dentaires chez ces patients (Bork et al. 2003).

Le Département de Médecine interne du CHU de Rouen nous adresse une jeune patiente de 22 ans pour bilan odontologique dans un contexte de refus de soins dentaires en raison de sa pathologie génétique : Déficit sévère en $\mathrm{C} 1 \mathrm{Inh}$ : $\mathrm{AOH}$ type I. Un premier bilan odontologique clinique et radiographique permet d'organiser avec le service de Médecine interne une prise en charge hospitalière en raison d'un état de polycaries $(35,47,28)$ et des foyers infectieux périapicaux $(14,46,36)$.

Pour chaque séance de soins, une hospitalisation de 48 heures est programmée et un traitement préventif est instauré a visé anti-œdémateuse.

Le protocole thérapeutique a consisté en l'administration préventive de Danatrol ${ }^{\circledR}(5 \mathrm{mg} / \mathrm{kg}$ sans dépasser $600 \mathrm{mg} /$ jour) 5 jours avant la séance de soins dentaires et poursuivie pendant 2 jours après le geste.

Ce protocole préventif ne pouvant garantir une protection absolue contre toute apparition d'angio-oedème, nous avions de plus sécurisé nos interventions par la mise en place d'un abord veineux et disposions immédiatement et à tout moment d'une solution injectable IV de conestat alfa, forme recombinante de l'inhibiteur C1 humain (Ruconest $\left.{ }^{\circledR} 2100 \mathrm{U}\right)$. La présence infirmière était systématique à chaque séance de soins odontologiques. L'originalité de ce cas clinique est de rappeler qu'au regard de la littérature, la prise en charge odontologique de ces patients ne peut être qu'hospitalière imposant une surveillance stricte des 48 heures post-opératoires et du recours éventuel à des médicaments à délivrance strictement hospitalière et administrables par voie endoveineuse (Van Sickels et al. 2010).

MOIZAN Hervé herve.moizan@gmail.com

This is an Open Access article distributed under the terms of the Creative Commons Attribution License 2.0, which permits unrestricted use, distribution, and reproduction in any medium, provided the original work is properly cited. 